\title{
Relações entre Estratégia Saúde da Família e Vigilância em Saúde na perspectiva de médicos de equipes de saúde da família em Minas Gerais, Brasil: uma análise qualitativa
} The relation between the Family Health Strategy and Health Surveillance from the perspective of family health team physicians in Minas Gerais, Brasil: a qualitative analysis

\author{
Gabrielly Cristiny Soares Silva ${ }^{a}$ \\ (D) https://orcid.org/0000-0003-3303-2394 \\ E-mail: gaby.crys24ळhotmail.com \\ Rosimár Alves Querinob \\ (D) https://orcid.org/0000-0002-7863-121। \\ E-mail: rosimar.querinoळuftm.edu.br \\ Rafaela Defendi Borges ${ }^{a}$ \\ (D) https://orcid.org/0000-0002-7335-143X \\ E-mail: defendi.rafaelaœgmail.com

\section{Giovanna Mendonça Ivanckoa} \\ (iD) https://orcid.org/0000-0003-3096-1868 \\ E-mail: giovannamedळhotmail.com \\ Maria Isabel Silva ${ }^{c}$ \\ (iD) https://orcid.org/0000-0002-6044-0097 \\ E-mail: fisiomisळyahoo.com.br

\section{Jean Ezequiel Limongi ${ }^{\mathrm{a}}$} \\ (iD) https://orcid.org/0000-0003-2649-9842 \\ E-mail: jeanlimongi®gmail.com \\ aUniversidade Federal de Uberlândia. Instituto de Geografia. \\ Curso de Graduação em Saúde Coletiva. Uberlândia, MG, Brasil. \\ buniversidade Federal do Triângulo Mineiro. Instituto de \\ Ciências da Saúde. Departamento de Saúde Coletiva. \\ Uberaba, MG, Brasil. \\ 'Universidade Federal de Uberlândia. Reitoria. Pró-Reitoria de \\ Graduação. Uberlândia, MG, Brasil.
}

\section{Correspondência}

Jean Ezequiel Limongi

Universidade Federal de Uberlândia. Av. João Naves de Ávila, 2121. Uberlândia, MG, Brasil. CEP 38408-100.

\section{Resumo}

Este estudo avaliou o conhecimento de médicos de equipes da Estratégia Saúde da Família (ESF) sobre a Vigilância em Saúde e suas áreas, de modo a compreender como se dá a relação entre estes setores e identificar os pontos positivos e os nós críticos que prejudicam a integração entre eles. Foi realizado grupo focal com oito médicos de municípios do interior de Minas Gerais. 0 tempo médio de atuação dos profissionais na ESF foi de 5,7 \pm 3,25 anos e o tempo médio de atuação no Sistema Único de Saúde foi de $6,8 \pm 3,33$ anos. Foram identificadas três categorias temáticas, quais sejam: "Compreensões sobre Vigilância em Saúde", "Vigilâncias Reconhecidas" e "Relações dos Profissionais da Atenção Básica com a Vigilância em Saúde”. A Vigilância em Saúde foi percebida como responsável pelo controle e prevenção de doenças e norteadora do planejamento das ações de saúde. As vigilâncias mais reconhecidas foram a Epidemiológica e Sanitária. Os principais nós críticos identificados estão associados à falta de conhecimento mútuo e interação entre a Atenção Básica e a Vigilância em Saúde, que por conseguinte leva ao pouco envolvimento e integração dessas áreas no processo de notificação, acompanhamento e resolução de doenças e agravos de responsabilidade compartilhada.

Palavras-chave: Estratégia Saúde da Família; Vigilância em Saúde; Atenção Básica; Pesquisa Qualitativa. 


\section{Introdução}

This study evaluated the knowledge of the Family Health Strategy team physicians about Health Surveillance and related areas to verify the relation between these sectors, allowing to identify the positive points and critical nodes that undermine the integration between them. A focus group was conducted with eight physicians from municipalities in the countryside of Minas Gerais. The average working time of professionals in FHS was 5.73 .25 years and the average working time in SUS was 6.8 3.33 years. Three thematic categories were identified, namely, "Understanding Health Surveillance", "Recognized Surveillance" and "Relation of Primary Care Professionals with Health Surveillance". Health Surveillance was perceived as responsible for disease control and prevention, as well as for guiding the planning of health actions. The most recognized types of vigilance was Epidemiological Surveillance and Sanitary Surveillance. The main critical nodes identified are associated with a lack of mutual knowledge and interaction between Primary Care and Health Surveillance, which consequently leads to the low involvement and integration of these areas in the process of notification, monitoring and resolution of diseases and grievances of shared responsibility.

Keywords: Family Health Strategy; Health Surveillance; Primary Health Care; Qualitative Research.
O Sistema Único de Saúde (SUS) foi criado pela Constituição Federal de 1988 para atender às diversas demandas em saúde da população. Para tanto, articulou-se diferentes setores, com finalidades específicas, seguindo o modelo de atenção à saúde. A Atenção Básica (AB) e a Vigilância em Saúde (VS) ocupam lugar estratégico no modelo de atenção ao favorecer a integralidade da atenção e fomentar a articulação dos níveis de serviço (Brasil, 2018a).

$\mathrm{A} A \mathrm{~B}$ se estrutura por meio de um conjunto de ações de saúde individuais, familiares e coletivas que promovem prevenção a doenças e proteção à saúde, com práticas de cuidado integrado e focadas em territórios específicos. Para isso, equipes multiprofissionais de saúde atuam com responsabilidade sanitária pelo seu território adscrito (Brasil, 2017).

AVS, na legislação brasileira vigente, se encarrega de um processo contínuo de coleta, consolidação, análise e disseminação de dados de saúde; ou seja, fornece subsídios para o planejamento e execução de medidas de promoção, prevenção e proteção à saúde (Brasil, 2013). Para tanto, a VS é organizada em diferentes áreas, que são interligadas entre si e que atuam como um eixo estruturante do SUS, cujo enfoque é epidemiológico, sanitário, ambiental, relacionado a saúde do trabalhador, promoção de saúde e situação de saúde (Teixeira et al., 2018).

Para que haja integralidade nas ações de saúde, atendendo às necessidades da população, é necessário que exista uma aproximação dos diversos setores, em uma perspectiva intra e intersetorial (Brasil, 2018b). Diversas estratégias foram elaboradas nas últimas décadas na saúde pública brasileira buscando integrar a $\mathrm{AB}$ e a VS, porém isto ainda constitui um dos grandes desafios do SUS em todas as esferas de gestão (Brasil, 2018b). Para que ocorra, os profissionais devem conhecer as responsabilidades, atribuições e objetivos não só de suas áreas de atuação, mas também de outras relacionadas, de modo a buscarem e fornecerem apoio uns aos outros e, até mesmo, saberem agir de maneira correta perante as situações que se depararem (Limongi; Menezes; Menezes, 2008). Embora a importância da atuação conjunta seja 
reconhecida, as ações ainda são executadas de forma fragmentada e pouco resolutiva e isto reflete diretamente na eficiência do SUS e no estado de saúde da população (Pinto; Pereira; Limongi, 2017). De forma operacional, VS e AB deveriam atuar utilizando a mesma base territorial, os mesmos indicadores a serem alcançados e sob gestão integrada (Limongi; Menezes; Menezes, 2008).

Nesse contexto, este estudo analisou o modo como médicos de equipes da Estratégia Saúde da Família (ESF) de municípios do interior de Minas Gerais compreendem a VS e as relações entre a ESF e a VS.

\section{Metodologia}

Trata-se de um estudo qualitativo desenvolvido com a técnica de grupo focal (GF) (Barbour, 2009; Minayo, 2017).

Participaram oito médicos que atuavam na ESF de munícipios distintos do interior de Minas Gerais, quais sejam: Patos de Minas, Uberlândia, Bom Despacho, Montes Claros e Araguari. Todos estes profissionais eram discentes de uma instituição federal de ensino, cursando mestrado profissional ofertado exclusivamente para médicos atuantes em ESF.

A caracterização dos participantes é descrita na Tabela 1. O tempo médio de atuação deles no SUS e na ESF teve pouca variação. Visando garantir sigilo e confidencialidade, cada participante foi identificado com a letra $\mathrm{P}$ seguida por um número.

\section{Tabela I - Caracterização dos profissionais médicos participantes da pesquisa $(n=8)$}

\begin{tabular}{ccccc} 
Participantes & Sexo & Idade & $\begin{array}{c}\text { Atuação } \\
\text { na ESF } \\
\text { (em anos) }\end{array}$ & $\begin{array}{c}\text { Atuação } \\
\text { no SUS } \\
\text { (em anos) }\end{array}$ \\
P1 & Feminino & 28 & 5 & 5 \\
P2 & Feminino & 30 & 4 & 4 \\
P3 & Masculino & 37 & 8 & 8 \\
P4 & Feminino & 26 & 3 & 8 \\
P5 & Masculino & 28 & 3 & 3 \\
P6 & Feminino & 40 & 5 & 7 \\
P7 & Masculino & 37 & 12 & 13 \\
P8 & Feminino & 53 & 5 & 5 \\
\hline
\end{tabular}

ESF: Estratégia Saúde da Família; SUS: Sistema Único de Saúde.
Dois encontros de GF foram conduzidos por dois moderadores com formações diferentes: uma pesquisadora formada em Ciências Sociais e com experiência em pesquisa qualitativa e um pesquisador com experiência de trabalho e pesquisa na VS. Contou, também, com participação de três bolsistas de iniciação científica na posição de observadoras, responsáveis por assistir e registrar as interações durante os GF.

Os encontros ocorreram nos dias 10 e 11 de maio de 2019 e tiveram duração média de 140 minutos. 0 local foi previamente definido pelos pesquisadores, garantindo aos participantes as condições de sigilo e privacidade. As gravações de áudio foram transcritas na íntegra para fins analíticos e descartadas ao término das análises. 0 primeiro GF teve início com questão disparadora que propunha uma reflexão sobre como os participantes definiriam "Vigilância em Saúde". Em seguida, os moderadores solicitaram que relatassem um exemplo do trabalho com a VS no seu cotidiano, em equipe de saúde e território de atuação. As perguntas também estimulavam os participantes a relatarem como a equipe atuaria em seu território e mobilizaria outras instituições/ setores para efetuar ações de VS.

Na sequência, foram empregadas duas situações problema e perguntas disparadoras elaboradas pela equipe e fundamentadas nas finalidades da pesquisa. Em cada encontro discutiu-se uma das situações, que constam nos quadros 1 e 2 .

As gravações de áudio foram transcritas na íntegra, sem inserções ou supressões, e submetidas a análise de conteúdo em modalidade temática seguindo as diretrizes estabelecidas por Minayo (2017) e Graneheim e Lundman (2004).

A pré-análise e a exploração do material foram realizadas por cada um dos integrantes da equipe separadamente. À luz das unidades de sentido e das categorias identificadas por cada pesquisador, foram avaliadas convergências e divergências. No caso das divergências, ocorreu nova leitura da transcrição para a finalização da análise. A análise de conteúdo temática resultou na construção das categorias e respectivas unidades de sentido, conforme Figura 1. 


\section{Quadro I - Situação Problema I - disparadora do I encontro de grupo focal}

Um município de Minas Gerais conta com a existência de apenas uma unidade de saúde da família (ESF), com três equipes para seus 35 mil habitantes, e não possui espaços adequados para prática de atividades físicas. Percebeu- se, ao longo dos anos, o crescimento populacional do município, devido à abertura de novos postos de trabalho. Pessoas oriundas da região Nordeste do Brasil, bem como da região norte mineira, são os principais migrantes. Uma família nordestina formada por Adriana ( 34 anos, boleira), Carlos ( 45 anos, desempregado) e seu filho Alexandre ( 5 anos, estudante) se mudou para o município e lá vivem há seis meses. Carlos apresenta um quadro de hepatoesplenomegalia moderada, palidez e emagrecimento, sugestivo de leishmaniose visceral; porém, devido à sua atual situação socioeconômica, não tem se preocupado tanto com isso. Adriana começou a vender seus bolos na rodoviária para complementar a renda da família. Ela acorda às $4 \mathrm{~h}$ da manhã para chegar na rodoviária às 5 h3o e começar a trabalhar. Como o mercado informal é imprevisível e nem todos os dias há uma boa venda, sobra bolo ao final do dia. Porém, a mercadoria que não é vendida na rodoviária, é consumida diariamente por ela, pelo marido e seu filho em casa. Além disso, Adriana é hipertensa e diante da rotina diária não consegue fazer o acompanhamento de seu tratamento com a equipe de saúde da família. Por vezes, Alexandre conta apenas com a alimentação que recebe na escola e os bolos feitos pela mãe. Ele começou a desenvolver um quadro de sobrepeso e problemas nutricionais. De forma geral, o estilo de vida das pessoas no bairro de Adriana e Carlos não é favorável para a saúde, com hábitos alimentares ruins, sedentarismo, tabagismo, alcoolismo e episódios de violência frequentes.

\section{Quadro 2 - Situação Problema 2 - disparadora do 2 encontro de grupo focal}

Uma unidade da Estratégia Saúde da Família foi recentemente inaugurada. Ela atende um território bastante heterogêneo, com grandes dimensões e caracterizado como urbano e parcialmente periurbano. Recentemente, foram notificados possíveis casos de Zika e Chikungunya, o que vem levando o município a rever o controle do Aedes Aegypti, que estava sendo satisfatório. No reconhecimento do território, os agentes comunitários de saúde (ACS) observaram com certa frequência nas residências o acúmulo de materiais sem utilidade, bem como condições de saneamento básico impróprias e irregulares. Além disso, em reunião da equipe de saúde da família, os ACS relataram como peculiaridade do território o trabalho informal nas residências. Em diversas residências há catadores de reciclados, que se multiplicaram com o desemprego. Em uma visita de rotina foi verificado este tipo de trabalho na fabricação de amaciantes de tecidos e desinfetantes (saneantes), no qual a mãe e os dois filhos, de 11 e 13 anos, trabalhavam 8 horas por dia. Em conversa com a ACS, a mãe relatou que o filho mais novo se sentiu mal no dia anterior, apresentando vômito e desorientação ao manipular uma das substâncias utilizadas para a fabricação dos amaciantes.

\section{Figura I - Categorias temáticas e unidades de sentido identificadas no estudo}

Vs: Vigilância em Saúde; AB: Atenção Básica.

\begin{tabular}{|c|c|}
\hline \multirow{5}{*}{ 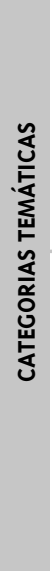 } & Compreensões sobre Vigilância em Saúde \\
\hline & $\begin{array}{l}\text { - VS como controle de doenças e agravos } \\
\text { - VS como prevenção } \\
\text { - } \quad \text { VS como norteadora do planejamento das ações em saúde }\end{array}$ \\
\hline & Vigilâncias reconhecidas \\
\hline & Relações dos profissionais da AB com a VS \\
\hline & $\begin{array}{l}\text { - Órgãos não se conhecem ou há profissionais específicos da } \\
\text { - } \quad \text { Comunicação deficiente } \\
\text { - } \quad \text { Notificações: erros de preenchimento, subnotificação } \\
\text { - Baixo envolvimento do médico de família na VS } \\
\text { - } \quad \text { Experiências de integração AB/VS: capacitação, participação } \\
\text { em reuniões }\end{array}$ \\
\hline
\end{tabular}


O referencial teórico-metodológico adotado foi a perspectiva dialética que permite compreender VS e AB como práticas históricas e sociais nas quais há uma relação dialética entre o agir e o pensar, entre os homens e destes com o mundo (Campos, 200o). Destarte, a forma como os médicos de ESF compreendem as relações entre a VS e AB revela como, em seu cotidiano, articulam dialeticamente teoria e prática de modo a produzir com as comunidades e territórios a atenção à saúde. É, também, este agir/pensar que permite a construção de suas percepções de mundo. Dado o caráter interdisciplinar do tema investigado, no processo interpretativo mobilizaram-se as produções acadêmica sobre ESF e VS.

O projeto foi submetido ao Comitê de Ética em Pesquisa da Universidade Federal de Uberlândia, com aprovação em $1^{\circ}$ de novembro de 2018 (Parecer $\mathrm{n}^{0}$ 2.996.099/2018). Os participantes da pesquisa assinaram e receberam uma cópia do Termo de Consentimento Livre e Esclarecido antes da coleta de dados.

\section{Resultados}

O tempo médio de atuação na ESF foi de 5,7 \pm 3,25 anos e o tempo médio de atuação no SUS foi de 6,8 \pm 3,33 anos. A idade média dos participantes foi de 32,28 $\pm 5,55$ anos, e a maioria era do sexo feminino (57,1\%).

Quanto às compreensões do termo VS, as definições fornecidas pelos médicos se fundamentaram em três unidades de sentido. 0 termo foi fortemente associado às ações de vigiar, monitorar, manter a segurança e evitar desfechos indesejáveis:

Vigilância é vigiar, é observar, é tá atento. Em saúde eu já penso, não só nos agravos, nas doenças, mas, também, nos determinantes de saúde. $\left(\mathrm{P}_{7}\right)$

Me vem em mente a palavra segurança [...] regras a serem seguidas baseadas em estudo. Tem algo de prevenir, de controlar a segurança, de justamente evitar sair do controle algumas situações, tanto na questão epidemiológica quanto na questão sanitária. É sempre registrar, estudar e agir em prol da segurança do tema que tá envolvido, seja o meio ambiente, seja o perfil epidemiológico, seja o sanitário. $\left(\mathrm{P}_{2}\right)$

Me remete muito às doenças, condições que a gente tem que vigiar de perto pra que elas não aconteçam de forma maior do que o esperado pra aquela população ali em geral. $\left(\mathrm{P}_{3}\right)$

Entenderos processos e evitar desfechos ruins. (P1)

Monitoramento também. (P2)

[pensar] nas doenças crônicas, no que é cadastrado, na importância de tá cadastrando os usuários diabéticos no SUS e pra promover ações sobre isso. ( $\left.\mathrm{P}_{5}\right)$

Houve o entendimento de VS como controle e preocupação não apenas com doenças e atividades curativistas, mas também com a identificação dos determinantes sociais de saúde e práticas preventivas, bem como a associação com ações de imunização:

As doenças crônicas não transmissíveis $e$ transmissíveis, principalmente. Então, a postura [...] de busca ativa também na questão de não só focar na prática curativista, mas na preventiva. Então, remete muito à prevenção. $(\mathrm{P} 1)$

Me veio à mente vacinação também, pra prevenção das doenças, ou então a cobertura vacinal, eu acho que é uma coisa que faz parte desse processo de vigilância. $\left(\mathrm{P}_{3}\right)$

Apesar da menção da VS como responsável pelo controle de agravos no âmbito sanitário e ambiental, a associação do termo com a notificação de agravos de ordem epidemiológica, principalmente com o controle das doenças de notificação compulsória transmissíveis e não-transmissíveis, foi a mais evidente:

pode ser doenças crônicas não transmissíveis [...], mas me remete muito às doenças transmissíveis que a gente tem lidado como a dengue, hanseníase. $\left(\mathrm{P}_{3}\right)$ 
Agente pensa muitonos agravos que são notificáveis, então doenças infecto-parasitárias de notificação compulsória ou não, os problemas do trabalho, tudo que é notificado. [...] Vigilância em Saúde eu acho que pode sermais amplo [...] pensar tambémno hipertenso, no diabético, nas doenças crônicas. $\left(\mathrm{P}_{5}\right)$

Também se caracterizou VS como um eixo norteador que subsidia o planejamento, tomadas de decisões e ações em saúde, pelo fato de dispor de dados e informações de saúde da população consolidados em sistemas de informação variados:

Notifica para a gente planejar as próximas ações. $\left(\mathrm{P}_{5}\right)$

[...] e na obtenção de dados também! Importante isso pra gente planejar a saúde. $(\mathrm{P} 1)$

[pensar] nas doenças crônicas, no que écadastrado, na importância de tá cadastrando os usuários diabéticos no SUS e pra promover ações sobre isso. $\left(\mathrm{P}_{5}\right)$

Em relação às áreas da VS reconhecidas, os participantes citaram todas, porém as mais referidas foram a Vigilância Epidemiológica (Vigep) e a Vigilância Sanitária (Visa):

evitar sair do controle em algumas situações, tanto na questão epidemiológica quanto na questão sanitária. $\tilde{E}$ sempre registrar, estudar e agir em prol de uma segurança do tema que tá envolvido, seja o meio ambiente, seja o perfil epidemiológico, seja o sanitário. $\left(\mathrm{P}_{2}\right)$

Quando me fala em VS me vem ações em saúde dentro da Vigilância Sanitária e dentro da Vigilância Epidemiológica. Sei que compõe, também, Saúde do Trabalhador e Vigilância do Meio Ambiente, mas eu acho que Vigilância Sanitária e Vigilância Epidemiológica são as ações principais que me remetem quando eu falo de Vigilância em Saúde. (P6)

Agente pensa muito nos agravos que são notificáveis. Então, doenças infecto-parasitárias de notificação compulsória ou não, problemas do trabalho, tudo que énotificado. ( $\left.\mathrm{P}_{5}\right)$

A gente fica muito mais com a parte das doenças de notificação, Vigilância Epidemiológica e muito superficial. $\left(\mathrm{P}_{7}\right)$

Gente, eunem sabia que tinha Vigilância Ambiental... (riso coletivo). Agente sabe, mas não sabe. Quando a gente conversa, a gente sabe que existe, mas assim, Vigilância Ambiental, Vigilância Sanitária, sabe aquela coisa, o que que cada umfaz, especificamente, eu não. $\left(\mathrm{P}_{3}\right)$

$\varepsilon$, não tem uma divisão certa, até onde vai a Vigilância Sanitária [...] onde vai a epidemiológica, onde vai o trabalho da equipe de saúde, não tem uma divisão certinha. $\left(\mathrm{P}_{3}\right)$

Como visto, a Vigilância em Saúde do Trabalhador (Visat) foi a área menos explorada, citada brevemente por apenas dois participantes. A Vigilância Ambiental em Saúde (Vigiam) foi referida como "Vigilância do Meio Ambiente" e com uma conotação voltada muitos mais para questões ambientais puramente, sem interface com a saúde. O desconhecimento da existência da área também foi relatado.

A referência à promoção da saúde também foi pouco abrangente, limitando-se a mencionar o intuito para além do curativismo e mais focado na prevenção, sem abarcar de fato a promoção: não só focar na prática curativista, mas na preventiva. Então, remete muito à prevenção $(\mathrm{P} 1)$.

A relação dos profissionais da $\mathrm{AB}$ com a VS foi o eixo temático mais explorado pelos participantes, sendo a maior parte dos relatos direcionados às dificuldades em se estabelecer um vínculo entre as duas esferas de atuação. A falta ou insuficiência da comunicação foi associada, em um primeiro momento, ao fato de os próprios médicos não se relacionarem com nenhum profissional da VS:

Eu não conheço ninguém da vigilância, quem faz essa comunicação é a enfermeira. Ela liga e fala "Ó tem tantas fichas, vem buscar fazendo um favor". ( $\left.\mathrm{P}_{4}\right)$ 
não passa por mim, eu não conheço ninguém da Vigilância, nunca fuina Vigilância, nunca ninguém teve uma convers a comigo, o que se passava era via enfermeiro, via coordenadordaAtenção [Básica]. $\left(\mathrm{P}_{2}\right)$

Eu tive isso de passarem os telefones [...] Só que, na prática, a gente não tinha um contato, mas eles passaram, a gente foi lá e eles passaram de cada setor o telefone pra gente entrar em contato. $\left(\mathrm{P}_{5}\right)$

Em seguida, os participantes a relacionaram ao próprio modo como se dá esta comunicação, de forma breve e descuidada, apenas para cobranças das fichas de notificação compulsória, sem que haja um entrosamento que permita de fato um diálogo entre os setores:

Lánarede[de unidade desaúde], umavezporsemana a moça da vigilância passava perguntando se tinha alguma coisa: "Tem alguma coisa pra gente?", "Não", ia embora. Então assim, era uma coisa de cinco minutos, então é uma comunicação muito falha. (P4)

Dá impressão ó: "Você escreve o papelzinho e deixa aqui com a gente”. Quer dizer, alguém mandou me falar isso, nem me ligou e nem foi pessoalmente me falar, então ó: "Notifica esse negócio da dengue que nós vamos resolver isso aípra lá depois, depois nós dá um comunicado e avisa vocês”. ( $\left(\mathrm{P}_{2}\right)$

pra encher os cadernos e pra receber as informações das notificações erradas, é só pra isso [...] que eles [da VS] procuram a gente. $\left(\mathrm{P}_{3}\right)$

As fichas vêm pra cáe aí eles ligam: "Ó tá tudo errado, táfaltando as coisas". [...] éuma comunicação muito ruim. (P4)

Apesar destes apontamentos, os participantes declararam a importância do contato e assumiram que eles mesmos se envolvem pouco com a VS, mesmo quando informados sobre as formas de comunicação:

a dificuldade de fazer essa comunicação com a Vigilância em si, porque tem que ter [...] essa comunicação [...] [precisa] saber o que que é, quem éo responsável, como a gente vai promover a ação. $\left(\mathrm{P}_{7}\right)$
Eu acho que tem culpa das duas partes, sabe. (P7)

Os participantes relataram dificuldades em reconhecer ações de VS como parte de suas atribuições. Além disso, reconheceram que a formação profissional médica tradicional não trabalha ações de saúde sob a perspectiva da VS e que são incentivados a resolverem problemas de saúde apenas tratando a doença.

Na minha formação tradicional, quando a gente pensa em Vigilância a gente imagina algo alheio à gente, só que na verdade, [...] a gente percebe que nós também no nosso dia-a-dia nós fazemos algumas ações que são de VS. (P1)

Eu não vivencio, parece que tá mais na televisão do que algo que é meu! Fico incomodado. $\left(\mathrm{P}_{2}\right)$

Eu acho que a gente se envolve pouco com a Vigilância. [...] Primeiro porque na nossa formação a gentenãoéestimulado a isso, a gente fica muito mais com a parte das doenças de notificação, Vigilância Epidemiológica e muito superficial. $\left(\mathrm{P}_{7}\right)$

A gente tá muito mais voltado pra doença do que a saúde, mesmo que a gente tenha esses espaços, batemos a tecla na doença, come bem, faz atividade física pra não ter diabetes, a gente nem faz grupo pra quem não tem problema de saúde. $\left(\mathrm{P}_{7}\right)$

Então, a gente táacabando que tá fazendo atividade de promoção individual. [...] eu acho que gente tem uma dificuldade por pressão mesmo externa mesmo [...] tem uma coisa que é da nossa própria formação, que a gente realmente foi formado pra tratar doença, não foi formado pra tratar de saúde. $\left(\mathrm{P}_{3}\right)$

Os participantes ainda consideraram que os médicos da ESF, quando comparados com profissionais de outras especialidades médicas, são mais articulados com a VS: Gente, isso a gente tá falando de Atenção Básica, de médico de família, de Estratégia Saúde da Família, imagina as outras especialidades médicas, isso aí passa longe $\left(\mathrm{P}_{7}\right)$.

Em relação às notificações, os médicos declararam que nem sempre se comprometem adequadamente 
com o preenchimento das fichas e reconheceram o impacto que isso gera para o planejamento das ações de saúde pela VS.

tá tendo muita dengue, a gente tánotificando muito [...] o preenchimento, às vezes eu não confiro todos os dados que os internos ou os residentes preenchem. Muitas notificações voltam. [...] às vezes a gente poderia ter corrigido o nosso, da equipe, mas eles também antes de pegar e trazer pra cá, pra ter comunicação. (P4)

Euvejo muito assim, eusousupervisor do Programa Mais Médicos, e eu vejo que os médicos não têm muita responsabilidade na alimentação do sistema de informação e, com isso, a própria vigilância perde um pouco da credibilidade em termos de número e informação, que aquiloérepassado pra ponta, para tomada de decisão. (P6)

Ele [o médico] não é comprometido com a inserção de informação da sua própria produção informa errado, informa com preguiça, e se ele não informa direito ele não dá muito crédito para a informação que chega, acho que issoéum ponto preocupante.(P6)

As subnotificações e fichas incompletas também foram justificadas pela falta de um sistema informatizado que permita o preenchimento da ficha concomitantemente ao momento em que se realiza a consulta.

o caso da informação, se tivesse um sistema pra digitar lána hora como a gente tem de produção. Acho que o tempo da informação e da resposta, acho que seria mais ágil, iria ajudarnessa comunicação. (P4)

Mas também por condições da assistência [...] a gente vê subnotificação, ficha incompleta. (P7)

Os participantes do grupo também relataram sua insatisfação com a falta de acompanhamento dos casos por eles notificados, questionando o seu papel apenas como notificadores, mas sem inteiração das ações que a VS promove após a notificação/ informação gerada.
Mas em VS uma coisa me incomoda um pouco, [...] por exemplo, veio um caso de dengue eu vou lá enotifico e tchau. Esse papel meu só de chegar e notificar, e daíque eu notifiquei? Nãovou participar do controle da dengue no meu bairro? [...] Dá impressão: "Você escreve os papeizinhos e deixa aqui com a gente”. [...] Ou seja, a gentenão tem uma participação muito direta pensandonessa questão dos órgãos. [...] Parece que nosso papel é só esse, ah veio uma pessoa com tosse há quatro semanas, "Ó enfermeira, veio um aqui", "Ahentão me dá isso aqui" etchau, acabou. (P2)

De modo contrário, um participante justificou a falta de acompanhamento dos casos pelo próprio desinteresse do médico no processo de notificação/ acompanhamento e, por conseguinte, no seu desfecho: Muitas vezes a gente preenche a ficha, mas não acompanha a investigação do caso, o que a gente poderia fazer também já que tá perto do paciente $\left(\mathrm{P}_{7}\right)$.

Ainda a respeito desta relação AB-VS, foram relatadas boas experiências de trabalho em conjunto com a equipe da VS e mencionou-se a presença do Agente de Controle de Endemias (ACE) nas unidades de saúde como uma peça importante e de grande valia na integração com a equipe de saúde da $\mathrm{AB}$, promovendo ações de saúde sob um olhar mais integral.

A gente tinha o Agente de Endemias e em épocas de surtos de dengue os Agentes de Endemias davam capacitações pros Agentes de Saúde, era bem legal. $\left(\mathrm{P}_{5}\right)$

A gente tinha a figura do Agente de Endemia no território, isso é muito bacana, e ele sempre participava da reunião junto com os Agentes Comunitários de Saúde, aívocêfalava: "-ó, naquela rua lá tá um acúmulo de lixo" [...] Então assim, era importantíssima a figura desse Agente de Endemia. $\left(\mathrm{P}_{7}\right)$

Na época da Chikungunya a gente tinha muita dúvida, não tinha protocolo [...] a gente não sabia como manejar e a vigilância sempremuita atenciosa [...] foi importantíssima [a parceria] porque eles construíram o protocolo de atendimento da Chikungunya dentro da secretaria municipal. $\left(\mathrm{P}_{7}\right)$ 


\section{Discussão}

$O$ estudo evidenciou que os setores VS e $A B$ parecem atuar de modo independente e com pouco envolvimento entre as partes. Foram observadas lacunas de conhecimento sobre a VS e pouca integração entre estas duas áreas. Tal dinâmica não é coerente com as diretrizes nacionais para a VS e a AB. A VS tem papel central na regulação, intervenção e atuação em condicionantes e determinantes da saúde com foco na proteção e promoção da saúde da população e na prevenção e controle de riscos, agravos e doenças (Brasil, 2018a). A interdisciplinaridade e intersetorialidade são características fundamentais para as práticas das ações de VS, que se estendem por todas as demais redes de atenção à saúde (Franco Neto et al., 2017). $\mathrm{AAB}$ é caracterizada por um conjunto de intervenções de saúde que organizam o seu processo de trabalho segundo o princípio da territorialidade, atendendo às necessidades de saúde da população adscrita, sendo este também o principal pilar da VS (Brasil, 2017).

Assim, o vínculo que a $\mathrm{AB}$ cria com a população desencadeia uma aproximação que permite entender melhor os problemas e necessidades de saúde, fazendo deste nível de atenção um lócus privilegiado para o desenvolvimento das ações de VS (Oliveira; Casanova, 2009).

Apesar de terem sido citadas definições coerentes sobre o termo VS, a dimensão ampliada deste conceito não foi devidamente explorada, visto que a principal referência de ações de vigilância citadas foram no âmbito da doença. Embora sejam identificados alguns avanços, a lógica assistencial, de caráter individual e curativista, ainda dirige grande parte das práticas das equipes de saúde do Brasil e foi identificada junto aos participantes do estudo. A cultura curativista impera, ainda, no consciente popular, e impede que ações voltadas à promoção e prevenção da saúde alcancem a eficiência necessária (Limongi; Menezes; Menezes, 2008).

De outro modo, o conceito ampliado de VS prevê a redefinição do seu objeto, propondo a saúde como o foco das ações e expandindo a compreensão do processo saúde-doença para além das limitações do modelo biomédico. Incorpora a dimensão dos determinantes dos problemas de saúde coletiva e individual e possibilita o desenvolvimento de um espaço da saúde, com atividades de promoção e prevenção, e não apenas de cuidados com a doença (Teixeira, 2004).

A área da VS mais reconhecida, tanto pela nomenclatura quanto pelas ações cotidianas, foi a Vigep. Isto pode ser explicado pelo longo histórico de ações de vigilância epidemiológica no país, além do fato de que o objeto de investigação desta área coincide em partes com os de ações médicoassistenciais, que estão relacionados às doenças e ações direcionadas a grupos ou problemas específicos, por exemplo, hipertensos, diabéticos, programas de controle da tuberculose, entre outros. Além disso, antes da década de 1940, as práticas de vigilância epidemiológica já eram difundidas, ainda que de modo descentralizado, tratando principalmente das doenças transmissíveis. Em 1970, foi criado o Sistema Nacional de Vigilância Epidemiológica. Destarte, desde a criação do SUS, outras dimensões e práticas da VS foram se desenvolvendo, ao passo que a Vigep já possuía infraestrutura e reconhecimento no âmbito das ações de saúde (Oliveira; Cruz, 2015).

As ações de vigilância sanitária também já existiam no Brasil desde o século XIX e, na década de 1970, tornaram-se mais visíveis ao setor da saúde com a criação da Secretaria Nacional de Vigilância Sanitária. Atualmente, ela está incorporada à Agência Nacional de Vigilância Sanitária (Anvisa) e possui ampla divulgação na mídia, o que também pode ter influenciado no maior reconhecimento entre os participantes, mesmo sem que práticas cotidianas fossem relatadas pelos profissionais da $\mathrm{AB}$ (Costa; Fernandes; Pimenta, 2008).

A cultura curativista, impregnada na saúde pública brasileira, faz com que questões propriamente relacionadas à saúde, como as ações de promoção à saúde, fiquem comprometidas, excluindo a parcela saudável da população do escopo de atuação da AB. Em geral existe a valorização ou priorização do evento já acontecido, contrariando um dos objetivos da vigilância, que é justamente o enfrentamento de riscos antes da instalação de determinado problema (Oliveira; Casanova, 2009). Este fato pôde ser evidenciado na fala dos participantes que descreveram muitas ações relacionadas às doenças e pouco se referiram a ações de promoção da saúde. 
A preocupação com os determinantes socioambientais de saúde faz parte das ações relacionadas à saúde ambiental e do trabalhador, que buscam compreender e mitigar os riscos e agravos advindos do ambiente de forma geral e do ambiente de trabalho (Dias et al., 2009). Era esperado que o espaço preferencial para estas vigilâncias fosse a $\mathrm{AB}$, já que as relações produção/trabalho/ambiente ocorrem nos territórios adscritos por este nível de atenção à saúde. No entanto, a incorporação das relações de produção-trabalho e saúde-ambiente na $\mathrm{AB}$ enfrenta grandes entraves para a sua efetivação. Como evidenciado neste estudo, muitas vezes os profissionais das ESF sequer conhecem estas vigilâncias ou as reconhecem de modo equivocado. É comum a confusão conceitual entre Saúde do Trabalhador e a Medicina do Trabalho, assim como entre a Vigilância em Saúde Ambiental e outros setores ligados essencialmente às temáticas ambientais. É importante considerar que muitos municípios de pequeno porte não possuem todas as áreas da VS distinguíveis no seu organograma, o que dificulta ainda mais o reconhecimento e a integração com a AB (Limongi et al., 2017).

Os profissionais médicos participantes relataram não haver qualquer contato direto deles com profissionais da VS. Este contato em geral ocorria via equipe de enfermagem ou por meio da coordenação da unidade de saúde. Ainda assim, foi também relatado que a comunicação entre a $\mathrm{AB}$ e a VS era breve, sem entrosamento e, geralmente, restrita às orientações sobre o preenchimento e recolhimento de fichas de doenças de notificação compulsória. O desconhecimento das ações realizadas pela VS também foi relatado. Este modus operandi da relação das unidades de saúde da família com a VS difere sobremaneira daquele preconizado. As ações de VS devem estar incorporadas no cotidiano das equipes da AB, em especial nas de Saúde da Família, assim como as equipes de VS devem trabalhar em parceria com as de AB (Noronha; Penna, 2007).

Assim, é fundamental que atribuições, saberes, práticas e objetivos de ambos campos de atuação sejam compartilhados e integrados, para que as necessidades de saúde da população sejam atendidas baseadas no princípio da integralidade (Libiano; Favoreto; Pinheiro, 2014).
A formação médica tradicional também se constitui como um fator limitante para o desenvolvimento de ações de vigilância na $\mathrm{AB}$, já que geralmente ela ocorre na perspectiva de valorização da especialidade e da superespecialidade em detrimento da formação com foco em saúde preventiva e promoção da saúde (Peixoto et al., 2019). Os participantes da pesquisa consideraram ser formados no "modelo tradicional" e alegaram grande distanciamento da saúde preventiva/vigilância em saúde durante a formação acadêmica Os profissionais de saúde não precisam necessariamente dominar todo o conhecimento sobre as diferentes esferas de atuação, mas devem ser capazes de ampliar e redefinir suas ações, por meio de um olhar interdisciplinar que não permite alienação do profissional ao próprio objeto de trabalho. As Diretrizes Curriculares Nacionais dos cursos de Medicina, instituídas em 2014, têm orientado as mudanças nos projetos políticos pedagógicos dos cursos para uma visão mais ampliada de saúde (Brasil, 2014).

Atualmente, a principal interação existente entre profissionais da $\mathrm{AB}$ com as equipes de VS se constitui no processo de notificação compulsória de doenças/agravos. Neste estudo, o tema foi amplamente abordado pelos participantes. Dois pontos merecem maior atenção, quais sejam, (1) o pouco envolvimento dos médicos com o processo de notificação e (2) o desestímulo no processo por não vislumbrarem continuidade nas ações e por não se sentirem como parte integrante delas.

O pouco envolvimento de médicos, assim como de outros profissionais na notificação obrigatória gera impactos importantes na vigilância e controle das doenças (Melo et al., 2018). Subnotificação, notificação tardia ou erros de preenchimento foram relatados pelos participantes do estudo, que se envolvem pouco com a notificação e por isso também não dão credibilidade aos dados públicos gerados a partir delas. Este ciclo vicioso compromete sobremaneira todo o sistema de saúde. A elevada demanda de atendimentos dos médicos e outros profissionais da linha de frente das unidades de saúde afeta todo o processo de notificação e acompanhamento dos casos. Profissionais designados para este fim, além de outras atribuições como o gerenciamento das unidades, divulgação e comunicação em saúde, 
que sobrecarregam as equipes de assistência, são fundamentais para a resolução destes velhos problemas no sistema de saúde pública brasileira. Os graduados em Saúde Pública/Saúde Coletiva, denominados como sanitaristas, são profissionais com perfil adequado para estas funções.

Considerando o segundo ponto, é importante destacar que existe a ideia entre profissionais de saúde de que a notificação compulsória é apenas uma atividade burocrática, sem ações advindas a partir dela. A deficiência na retroalimentação por parte das equipes de vigilância é o principal motivo deste pensamento e impede que os profissionais da $\mathrm{AB}$ reconheçam sua contribuição no processo. A retroalimentação é um dos pilares do sistema de VS preconizado pelo Ministério da Saúde. É fundamental a devolução de informações aos níveis de atenção geradores dos dados iniciais, desde dados específicos relativos à notificação gerada, até a comunicação de uma situação epidemiológica mais complexa que esteja ocorrendo em determinada região (Brasil, 2005).

O Ministério da Saúde brasileiro fez inúmeras tentativas de integração da VS e a AB, sendo que em 2008 este objetivo foi assumido como prioridade político-institucional. Neste sentido, recentemente foram propostas diretrizes para que sejam aplicadas em diferentes níveis de gestão no país. Dentre estas, a organização do processo de trabalho integrado e a utilização de território único para VS e a $\mathrm{AB}$ são as mais emblemáticas (Brasil, 2018a, 2018b). Os achados deste estudo, que demonstram a falta de convergência e sincronicidade das ações da $\mathrm{AB}$ e VS, justificam a urgente necessidade da aplicação destas diretrizes. A VS e a AB possuem atribuições em caráter de corresponsabilidade, por isso é imperativo que ações de VS sejam descentralizadas para a AB, inclusive com o oferecimento de serviços da primeira na segunda. Exemplos estimulantes e exitosos foram relatados, como a diminuição de casos de malária no norte do país em $50 \%$ devido principalmente à integração das equipes de controle de endemias (VS) e AB (Ladislau, 2007). Este tipo de parceria foi relatado positivamente neste estudo, mesmo que em contextos diferentes, confirmando a eficiência das ações conjuntas.

As especialidades da VS são complexas, interrelacionadas e estão em constante alteração, sendo o seu domínio desafiador até mesmo para os profissionais da vigilância. Assim, a educação permanente em saúde se mostra uma excelente alternativa de educação continuada para os profissionais de saúde, permitindo identificar problemas no trabalho cotidiano e propondo a integração de saberes e práticas interdisciplinares (Gigante; Campos, 2016).

\section{Considerações finais}

É possível promover a articulação da VS e AB, respeitando as particularidades de cada esfera de atuação e considerando a AB como agente de vigilância no território em que atua, sendo capaz de identificar riscos aos quais a comunidade está exposta, com acionamento correto dos setores da vigilância, em uma perspectiva de corresponsabilidade para solucionarem os problemas, garantindo à população o cuidado integral à saúde.

Um dos principais obstáculos para a implementação de ações intersetoriais entre $A B$ e VS advém da tradicional dicotomia vigilânciaassistência na oferta de serviços de saúde no Brasil, que desde o passado privilegiou o modelo hospitalar e curativo, centrado na doença, em detrimento às ações de cunho preventivo. Assim sendo, é preciso superar esta lógica assistencial de caráter individual para que a atuação da $\mathrm{AB}$ sob a perspectiva da VS se concretize, evitando práticas pontuais e fragmentadas que originam um sistema de saúde com ações descontextualizadas e pouco resolutivas (Campos, 2003).

O tema aqui abordado é pouco explorado e, por isso, não existem referências compatíveis para se fazer comparações com outras regiões, sendo esta uma limitação importante deste estudo. Esta abordagem em outras localidades do país, principalmente neste momento de incentivo governamental para a integração das áreas de $A B$ e VS, é pertinente.

Por fim, é necessário reconhecer que diversas estratégias foram implementadas pelo nível de gestão federal e as iniciativas de integração da AB e VS devem ser agora operacionalizadas nos estados e municípios, considerando as especificidades locais. 


\section{Referências}

BARBOUR, R. Grupos focais. Porto Alegre: Artmed, 2009.

BRASIL. Ministério da Saúde. Secretaria de Vigilância em Saúde. Guia de vigilância epidemiológica. 6. ed. Brasília, DF, 2005.

BRASIL. Ministério da Saúde. Portaria GM $\mathrm{n}^{0} 1.378$, de 9 de julho de 2013. Regulamenta as responsabilidades e define diretrizes para execução e financiamento das ações de Vigilância em Saúde pela União, Estados, Distrito Federal e Municípios, relativos ao Sistema Nacional de Vigilância em Saúde e Sistema Nacional de Vigilância Sanitária. Diário Oficial da União, Brasília, DF, 9 jul. 2013. Disponível em: <https://bit.ly/2ZpEgCs>. Acesso em: 27 nov. 2019.

BRASIL. Ministério da Educação. Resolução $\mathrm{n}^{0}{ }_{3}$, de 20 de junho de 2014. Institui diretrizes curriculares nacionais do curso de graduação em medicina e dá outras providências. Diário Oficial da União, Brasília, DF, 23 jun. 2014.

BRASIL. Ministério da Saúde. Portaria nº 2.436, de 21 de setembro de 2017. Aprova a Política Nacional de Atenção Básica, estabelecendo a revisão de diretrizes para a organização da Atenção Básica, no âmbito do Sistema Único de Saúde (SUS). Diário Oficial da União, Brasília, DF, 22 set. 2017 . Seção 1, p. 68.

BRASIL. Ministério da Saúde. Conselho Nacional de Saúde. Resolução MS/CNS nº 588, de 12 de julho de 2018. Fica instituída a Política Nacional de Vigilância em Saúde (PNVS), aprovada por meio desta resolução. Diário Oficial da União, Brasília, DF, 13 ago. 2018a. Seção 1, p. 87.

BRASIL. Ministério da Saúde. Secretaria de Atenção à Saúde. Secretaria de Vigilância em Saúde. Guia Política Nacional de Atenção Básica Módulo 1: integração Atenção Básica e Vigilância em Saúde. Brasília, DF, 2018b. Disponível em: <https://bit.ly/2BWIOYU>. Acesso em: 27 nov. 2019.

CAMPOS, C. E. A. O desafio da integralidade segundo as perspectivas da vigilância da saúde e da saúde da família. Ciências \& Saúde Coletiva, Rio de Janeiro, v. 8, n. 2, p. 596-584, 2003.
CAMPOS, G. W. S. Saúde pública e saúde coletiva: campo e núcleo de saberes e práticas. Ciência \& Saúde Coletiva, Rio de Janeiro, v. 5, n. 2, p. 219-230, 2000.

COSTA, E. A.; FERNANDES, T. M.; PIMENTA, T. S. A vigilância sanitária nas políticas de saúde no Brasil e a construção da identidade de seus trabalhadores (1976-1999). Ciência \& Saúde Coletiva, Rio de Janeiro, v. 13, n. 3, p. 995-1004, 2008.

DIAS, E. C. et al. Saúde ambiental e saúde do trabalhador na atenção primária à saúde, no SUS: oportunidades e desafios. Ciência \& Saúde Coletiva, Rio de Janeiro, v. 14, n. 6, p. 2061-2070, 2009.

FRANCO NETO, G. F. et al. Vigilância em Saúde brasileira: reflexões e contribuição ao debate da $1^{a}$ Conferência Nacional de Vigilância em Saúde. Ciência e Saúde Coletiva, Rio de Janeiro, v. 22, n. 10 p. 3137-3148, 2017.

GIGANTE, R. L.; CAMPOS, G. W. S. Política de Formação e Educação Permanente em Saúde no Brasil: bases legais e referências teóricas. Trabalho, Educação e Saúde, Rio de Janeiro, v. 14, n. 3, p. 747-763, 2016.

GRANEHEIM, U. H.; LUNDMAN, B. Qualitative content analysis in nursing research: concepts, procedures and measures to achieve trustworthiness. Nurse Education Today, Edimburgo, v. 24, n. 2, p. 105-112, 2004.

LADISLAU, J. L. B. Agentes comunitários de Saúde para combater a malária no norte do país. Revista Brasileira Saúde da Família, Brasília, DF, ano VIII, n. 16, p. 46-49, 2007.

LIBIANO, K. R.; FAVORETO, C. A. O.; PINHEIRO, R. Análise da integração da Vigilância Ambiental no controle da dengue com a Estratégia Saúde da Família: impacto nos saberes e práticas dos agentes comunitários de saúde. Physis, Rio de Janeiro, v. 24, n. 1, p. 147-163, 2014.

LIMONGI, J. E.; MENEZES, E. C.; MENEZES, A. C. Vigilância em Saúde no Programa Saúde da Família. Hygeia, Uberlândia, v. 4, n. 7, p. 35-44, 2008.

LIMONGI, J. E. et al. Estrutura e processos da Vigilância em Saúde em municípios mineiros: uma análise qualiquantitativa. Cadernos Saúde Coletiva, Rio de Janeiro, v. 25, n. 1, p. 31-44, 2017. 
MELO, M. A. S. et al. Percepção dos profissionais de saúde sobre os fatores associados à subnotificação no Sistema Nacional de Agravos de Notificação. Revista de Administração em Saúde, São Paulo, v. 18, n. 71, p. 1-17, 2018.

MINAYO, M. C. S. O desafio do conhecimento: pesquisa qualitativa em saúde. 15. ed. São Paulo: Hucitec, 2017.

NORONHA, J. C.; PENNA, G. O. Saúde da Família e Vigilância em Saúde: em busca da integração das práticas. Revista Brasileira Saúde da Família, Brasília, DF, ano VIII, n. 16, p. 4-9, 2007. Entrevista concedida a Patrícia Álvares.

OLIVEIRA, C. M.; CASANOVA, A. O. Vigilância da saúde no espaço de práticas da atenção básica.

Ciência e Saúde Coletiva, Rio de Janeiro, v. 14, n. 3 , p. 929-936, 2009.

OLIVEIRA, C. M.; CRUZ, M. M. Sistema de

Vigilância em Saúde no Brasil: avanços e desafios.
Saúde em Debate, Rio de Janeiro, v. 39, n. 104, p. $255-267,2015$.

PEIXOTO, M. T. et al. Formação médica na Atenção Primária à Saúde: experiência com múltiplas abordagens nas práticas de integração ensino, serviço e comunidade. Interface, Botucatu, v. 23, p. e170794, 2019. Suplemento 1.

PINTO, D. S.; PEREIRA, B. B.; LIMONGI, J. E. Avaliação do conhecimento sobre Vigilância em Saúde entre os profissionais do Sistema Único de Saúde, Uberlândia, Minas Gerais. Journal of Health \& Biological Sciences, Fortaleza, v. 5, n. 1, p. 37-43, 2017.

TEIXEIRA, C. F. Saúde da família, promoção e vigilância da saúde: construindo a integralidade da Atenção à Saúde no SUS. Revista Brasileira de Saúde da Família, Brasília, DF, v. 5, n. 7, p. 10-23, 2004.

TEIXEIRA, M. G. et al. Health surveillance at the SUS: development, effects and perspectives. Ciência e Saúde Coletiva, Rio de Janeiro, v. 23, n. 6 p. 1811-1818, 2018.

\section{Contribuição dos autores}

Todos os autores participaram efetivamente na concepção e no delineamento do projeto, na análise e interpretação dos dados, na redação do artigo, na revisão crítica e na aprovação da versão a ser publicada.

Recebido: $29 / 04 / 2020$

Aprovado: 20/06/2020 